\section{Fibrose angiocêntrica eosinofílica da cavidade nasal: relato de caso e revisão de literatura}

\author{
Roberto Alcântara Maia ${ }^{1}$, Gilson Araujo Castro ${ }^{2}$
}

Resumo / Summary

\section{Eosinophilic angiocentric fibrosis of the nasal cavity: case report and literature review}

Palavras-chave: fibrose angiocêntrica eosinofílica, obstrução nasal, cavidade nasal.

Key words: eosinophilic angiocentric fibrosis, nasal obstruction, nasal cavity.

\begin{abstract}
$\mathbf{A}$
fibrose angiocêntrica eosinofilica (FAE) é uma doença rara que acomete o trato nasossinusal. Poucos casos foram relatados até o momento na literatura, sendo o acometimento, em geral, na cavidade nasal e nos seios maxilares, e menos comumente, na região subglótica. A doença acarreta uma proliferação fibrótica da mucosa nasal com sintomas nasais obstrutivos. Apresentamos o caso de um paciente de 62 anos, sexo masculino, com obstrução nasal de longa data. Ao exame físico apresentava um alargamento importante da porção anterior do septo, com obstrução bilateral da cavidade nasal. O paciente foi submetido a septoplastia e o material obtido do septo foi encaminhado para exame histopatológico, sendo feito o diagnóstico de FAE. O objetivo deste relato de caso é alertar os otorrinolaringologistas sobre esta rara doença, seu diagnóstico diferencial em relação as doenças granulomatosas nasais, seus aspectos histopatológicos e clínicos.
\end{abstract}

\begin{abstract}
E Oosinophilic angiocentric fibrosis (EAF) is a rare disease which affects the sinonasal tract. A few cases have been reported in the literature affecting the nose and maxillary sinus with even fewer cases in the subglottic area. The disease causes fibrotic proliferation of the nasal mucosa and nasal obstructive symptoms. We present the case of a male patient, 62 years old, with history of nasal obstruction for several years. The clinical examination showed septal enlargement and bilateral nasal obstruction. The patient was submitted to a septoplasty and the septal material was sent for histological examination, which showed the diagnosis of EAF. The aim of this report is to show a rare benign lesion (EAF) associated with nasal obstruction, its differential diagnosis with nasal granulomatosis and its histopathological and clinical features.
\end{abstract}

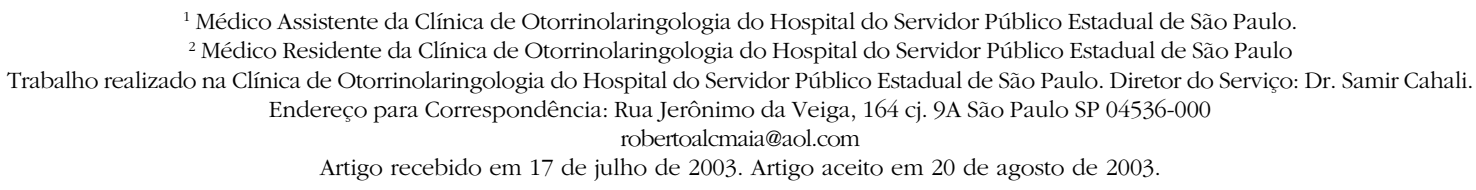

${ }^{1}$ Médico Assistente da Clínica de Otorrinolaringologia do Hospital do Servidor Público Estadual de São Paulo. ${ }^{2}$ Médico Residente da Clínica de Otorrinolaringologia do Hospital do Servidor Público Estadual de São Paulo Trabalho realizado na Clínica de Otorrinolaringologia do Hospital do Servidor Público Estadual de São Paulo. Diretor do Serviço: Dr. Samir Cahali. Endereço para Correspondência: Rua Jerônimo da Veiga, 164 cj. 9A São Paulo SP 04536-000 robertoalcmaia@aol.com

Artigo recebido em 17 de julho de 2003. Artigo aceito em 20 de agosto de 2003. 


\section{INTRODUÇÃO}

A fibrose angiocêntrica eosinofílica (FAE) é uma afecção rara que acomete o trato nasossinusal de indivíduos jovens, em geral mulheres, levando à proliferação fibrótica da mucosa nasal e sintomas obstrutivos de vias aéreas superiores. Menos comumente a doença também pode acometer a região subglótica da laringe. Esta condição foi descrita inicialmente por Roberts e McCann em 1985, que notaram uma doença de etiologia desconhecida afetando a mucosa nasal em dois pacientes e a região da subglote de um terceiro paciente. Estes autores acreditaram tratar-se de uma variante do granuloma facial ${ }^{1}$. Na literatura existe até o momento, para nosso conhecimento, apenas 13 casos relatados, sendo nove em mulheres e quatro em homens.

Histologicamente, a FAE se caracteriza por um infiltrado perivascular rico em eosinófilos, plasmócitos e histiócitos que progride para fibrose concêntrica perivascular. De modo diferente ao que acontece nas doenças granulomatosas nasais, como a granulomatose de Wegener, sarcoidose e tuberculose, dentre outras, os processos de vasculite necrotizante, necrose fibrinóide, trombose intravascular ou formações de granulomas aqui estão ausentes ${ }^{1}$. O processo de fibrose acometendo o septo nasal termina por levar a obstrução nasal, ao passo que o acometimento da laringe pode causar estenose subglótica ${ }^{2}$.

A doença nasal acomete tanto a mucosa do septo como a da parede lateral da cavidade nasal, o que também ajuda a diferenciar a FAE das doenças granulomatosas de linha média, que tendem a poupar a parede lateral. Além disso, na FAE não ocorrem ulcerações mucosas como nas granulomatoses ${ }^{2}$.

Indivíduos com FAE não têm acometimento sistêmico. Devido ao importante infiltrado eosinofílico perivascular existente foi sugerida a possibilidade de a sua causa ser de natureza alérgica, o que não tem comprovação até o momento. Diversas tentativas terapêuticas já foram feitas para esta doença, mas a maioria dos autores cita a cirurgia como o tratamento de escolha, embora possa haver múltiplas recorrências. Deste modo, algumas formas de tratamento coadjuvante foram tentadas, no sentido de tentar prevenir as recorrências e melhorar o controle clínico da doença, tais como o uso de corticosteróides por infiltração local ou via sistêmica. Nenhuma das formas de tratamento clínico descritas demonstraram bons resultados. A FAE permanece, até o momento, como uma doença de tratamento controverso ${ }^{3,4}$.

\section{APRESENTAÇ̃̃o DO CASO CLÍNICO}

Paciente H.P.A., 62 anos, sexo masculino, com queixa de obstrução nasal bilateral de longa data; negava queixas álgicas, episódios de sinusites ou epistaxes. Apresentava ao exame físico um desvio septal acentuado para direita além de espessamento significativo da região septal anterior, comprometendo a permeabilidade nasal bilateralmente; a nasofibroscopia mostrou a presença de um pólipo de aspecto benigno, bloqueando o meato médio direito.

Os seguintes exames subsidiários foram realizados:

1- Tomografia computadorizada de seios paranasais que mostrava desvio e espessamento septal, associado a velamento difuso de seio maxilar direito com bloqueio do complexo ostiomeatal do mesmo lado (Figura 1).

2- Exames de sangue laboratoriais: hemograma e velocidade de hemosedimentação normais; dosagem de IgA, IgG, IgM normais sendo a IgE significativamente aumentada; IgE específica (RAST) para pó caseiro não detectável; dosagem de anticorpos anti-citoplasma de neutrófilo (ANCA) não reativo; reação cutânea pelo PPD não reator.

O paciente foi submetido a procedimento cirúrgico nasal onde se observou septo cartilaginoso anterior bastante espessado, com aspecto de tecido fibrótico. Foi realizado o descolamento da mucosa com o septo nasal e feita a remoção de boa parte da estrutura fibrótica do septo para redução da espessura do mesmo. Realizou-se também, por via endoscópica, a uncinectomia direita, polipectomia e etmoidectomia anterior direita.

O material removido do septo nasal foi enviado para exame histopatológico cujo relatório revelou: "Tecido conjuntivo denso com focos de reação inflamatória crônica linfoplasmocitária e eosinofílica. Este tecido por vezes permeia o tecido cartilaginoso adjacente e exibe focos de calcificação. Em áreas notam-se vasos sangüíneos com fibrose concêntrica com aspectos de casca de cebola, permeados por infiltrado inflamatório rico em eosinófilos" (Figura 2).

O paciente evoluiu bem no pós-operatório, sem maiores complicações, exceto pela formação de crostas nasais que cederam com lavagens de soro fisiológico por cerca de 30 dias. No médio prazo, apresentou discreto sangramento nasal que melhorou após a suspensão temporária de medicação tópica contendo corticosteróides. Num período de acompanhamento pós-operatório de 24 meses não apresentou sinais de recidiva do espessamento septal nem dos pólipos nasais.

\section{DISCUSSÃO}

A fibrose angiocêntrica eosinofilica é uma doença rara em que ocorre fibrose submucosa do trato sinonasal e menos comumente da região subglótica da laringe $\mathrm{e}^{1,5}$. Os pacientes acometidos por esta afecção podem apresentar massa na cavidade nasal, dor maxilar, epistaxe, sinusites de repetição, rinorréia, ou mais comumente, sintomas nasais obstrutivos de longa duração ${ }^{3,6}$. Os autores que inicialmente descreveram esta doença acreditaram tratar-se de uma variante do granuloma facial, o que não é consenso na literatura ${ }^{1}$. Para 


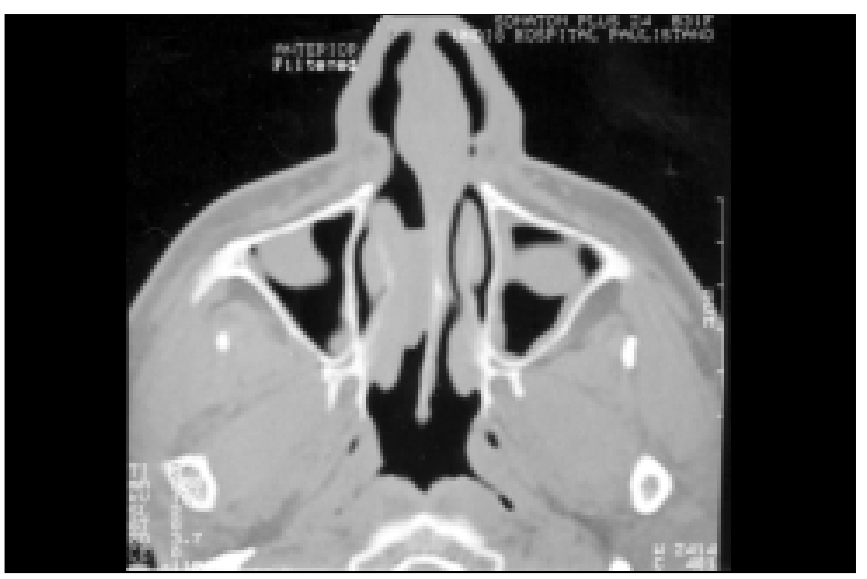

Figura 1. Corte axial de tomografia computadorizada de face

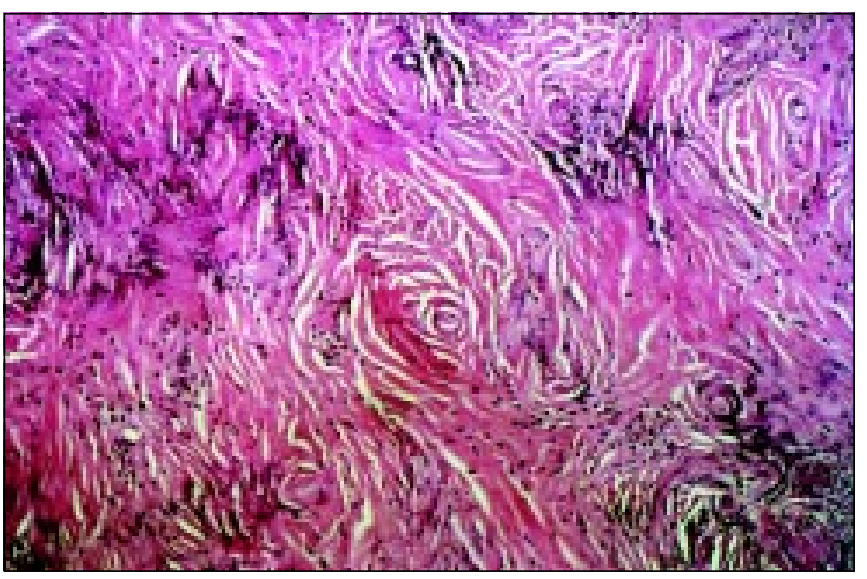

Figura 2. Exame hispatológico de mucosa de septo nasal

nosso conhecimento, existem apenas 13 casos descritos na literatura médica mundial até o presente momento. A FAE apresenta uma predileção por mulheres jovens e de meiaidade com uma razão de incidência entre o sexo masculino e o feminino de 4/9, com idades variando de 25 a 64 anos, sendo a média das idades de 44,6 anos.

A etiologia da FAE não é conhecida. A presença de eosinófilos nas lesões e a história clínica de rinite alérgica em pelo menos seis dos pacientes já relatados sugere uma possível causa alérgica ${ }^{7}$. Trauma nasal prévio, inclusive por cirurgia septal, foi aventado por Matai et al. ${ }^{7}$. Thompson et al. ${ }^{3}$, contudo, relataram três casos sem associação com alergia ou trauma nasal. Deste modo, a associação com doenças alérgicas ou trauma nasal pode ser mera coincidência. Também não há, até o momento, associação da FAE com polipose nasal ${ }^{1-9}$.

O diagnóstico da FAE pode ser suspeitado em pacientes com história de obstrução nasal de longa duração e que apresentam massa na cavidade nasal (na localização do septo ou da cartilagem lateral principalmente). Este aspecto pode ser visualizado como alargamento septal em exames de imagem. A confirmação diagnóstica, todavia, só pode ser feita através de exame histopatológico ${ }^{7,8}$.

O exame histológico mostra lesões em diferentes estágios de evolução, desde um processo inflamatório inicial com proliferação vascular e um denso infiltrado de linfócitos, plasmócitos, neutrófilos e principalmente eosinófilos, até áreas de fibrose perivascular com espessamento da parede dos vasos por proliferação de fibroblastos ${ }^{3,8}$. Não existe reação granulomatosa, processo de necrose vascular ou presença de folículos linfóides ${ }^{8}$. O exame de imunohistoquímica não é obrigatório para o diagnóstico, mas quando realizado, confirma a natureza inflamatória da FAE, diferenciando-a de neoplasias?

Quanto aos exames de imagem, a radiografia simples mostra aspectos inespecíficos como opacificação dos seios paranasais, esclerose óssea e destruição focal de tecidos ósseos da cavidade nasal ou da parede do seio maxilar. A tomografia computadorizada pode ser utilizada para avaliar a extensão das lesões, tanto nasais quanto laríngeas, assim como o grau de envolvimento das estruturas anatômicas ${ }^{7}$.

O diagnóstico diferencial da FAE inclui a granulomatose de Wegener, doença de Kimura, hiperplasia angiolinfóide, tumor miofibroblástico (pseudotumor inflamatório), granuloma facial, síndrome de Churg-Strauss, sarcoidose, síndrome de Sjogren, doenças granulomatosas infecciosas como a tuberculose e a hanseníase, além de neoplasias neurogênicas (schwannoma), vasculares (angiofibroma) e mesenquimais (fibroma, fibrosarcoma, fasciite nodular, fibromatose $)^{1-9}$. Para Thompson et al. $^{3}$, os principais diagnósticos diferenciais seriam granulomatose de Wegener, síndrome de Churg-Strauss e fibrossarcoma. As diferentes manifestações clínicas combinadas com os achados histológicos, laboratoriais e imunohistoquímicos ajudam a diferenciar estas doenças entre $\mathrm{si}^{3}$.

Deste modo alguns exames poderiam ser solicitados nestes pacientes com suspeita de doenças granulomatosas nasais: contagem diferencial de células sangüíneas (eosinofilia importante é encontrada na síndrome de Churg-Strauss); velocidade de hemossedimentação (que está aumentado nas granulomatoses e nas colagenoses, podendo estar normal na FAE); anticorpos contra citoplasma de neutrófilos - ANCA (podem estar aumentados na granulomatose de Wegener); radiografia simples de tórax para investigar sarcoidose e tuberculose; reação de Mantoux - PPD para tuberculose. Além disso, no sentido de pesquisar quadro alérgico poderiam ser solicitados dosagem de IgE sérica e ensaio radioimunoenzimático (RAST) para detectar anticorpos IgE específicos contra antígenos domésticos e fúngicos ${ }^{1-9}$.

Histologicamente, a maioria das lesões podem ser diferenciadas entre si. Na síndrome de Churg-Strauss ocorre necrose fibrinóide dos vasos, além da presença de granulomas. Na granulomatose de Wegener ocorre vasculite necrotizante, trombose intravascular e formação de 
granulomas acometendo, além do trato nasossinusal, outros sítios como os rins e os pulmões ${ }^{2,3}$. Loane et al. ${ }^{9}$ relataram o caso de um paciente com granulomatose de Wegener e FAE simultaneamente, sugerindo um possível mecanismo semelhante na patogênese das duas doenças. A doença de Kimura e o pseudotumor inflamatório podem ser distintos da FAE pelo fato de que nestas lesões o infiltrado inflamatório é misto e não há fibrose importante, além disso, na doença de Kimura ocorrem densos agregados linfóides com centros germinativos bem distintos. No granuloma facial, por outro lado, ocorre infiltrado inflamatório polimorfo com vasculite freqüente levando a trombose e necrose fibrinóide da parede dos vasos, além de extravasamento extravascular de eritrócitos. De todos os casos relacionados na literatura em apenas dois houve apresentação simultânea do granuloma facial com a FAE. Na FAE ocorre uma característica fibrose perivascular concêntrica, que não é encontrada em outras patologias, além de que estão ausentes necrose vascular e granulomas com células gigantes ${ }^{3,6}$.

O tratamento da FAE é principalmente cirúrgico para a maioria dos autores, podendo ser necessárias várias intervenções cirúrgicas, dado o caráter recidivante da doença ${ }^{1-10}$. Corticosteróides por via tópica ou sistêmica ou dapsona têm sido usados como terapia coadjuvante, mas sem resultados satisfatórios ${ }^{1-10}$. Agentes citotóxicos não foram usados até o momento, e não sabemos deste modo se podem trazer algum benefício no controle da doença ${ }^{3}$.

\section{COMENTÁRIOS FINAIS}

Este relato de caso procura alertar os otorrinolaringologistas sobre esta rara afecção. A FAE passa a figurar no diagnóstico diferencial das doenças granulomatosas e neoplásicas nasais. Trata-se de uma doença de curso insidioso que acaba por acarretar fundamentalmente sintomas obstrutivos de vias aéreas superiores. O diagnóstico definitivo é fundamentado nos aspectos histológicos. Até o momento, o único tratamento preconizado é essencialmente cirúrgico, embora seja conhecido seu aspecto recidivante pós cirurgia.

\section{AGRADECIMENTO}

Nossos agradecimentos ao Dr. Emílio M. Pereira, médico patologista do Laboratório Salomão e Zoppi pelo estudo histopatológico realizado neste caso.

\section{REFERÊNCIAS BIBLIOGRÁFICAS}

1. Roberts PF, McCann BG. Eosinophilic angiocentric fibrosis of the upper respiratory tract: a mucosal variant of granuloma faciale? A report of three cases. Histopathology 1985; 9: 1217-25.

2. Burns BV, Roberts PF, Path FRC et al. Eosinophilic angiocentric fibrosis affecting the nasal cavity. A mucosal variant of the skin lesion granuloma faciale. J Laryngol Otol 2001; 115: 223-6.

3. Thompson LD, Heffner DK. Sinonasal tract Eosinophilic angiocentric fibrosis. Am J Clin Pathol. 2001; 115(2): 243-8.

4. Roberts PF, McCann BG. Eosinophilic angiocentric fibrosis of the upper respiratory tract: a postcript. Histopathology 1997; 3: 385-6.

5. Fageeh NA, Mai KT, Odell PF. Eosinophilic angiocentric fibrosis of the subglottic region of the larynx and upper trachea. J Otolaryngol 1996; 25(4): 276-8.

6. Pereira EM, Millas I, Reis-Filho JS et al. Eosinophilic angiocentric fibrosis of the sinonasal tract: report on the clinicopathologic features of a case and review of the literature. Head Neck 2002; 24(3): 307-11.

7. Matai V, Baer S, Barnes S, Boxer M. Eosinophilic angiocentric fibrosis. J Laryngol Otol 2000; 114(7): 563-4.

8. Loane J, Jaramillo M, Young HÁ, Kerr KM. Eosinophilic angiocentric fibrosis and Wegener's granulomatosis: a case report and literature review. J Clin Pathol 2001; 54(8): 640-1.

9. Owa AO, Boyle S, Gallimore AP. Eosinophilic angiocentric fibrosis as a cause of nasal obstruction. Rhinology 2002; 40: 41-3.

10. Altemani AM, Bem ZP, Sakano E, Altemani JM. Eosinophilic angiocentric fibrosis of the nasal cavity. Mod Pathol 1997; 10(4): 391-3. 